Classification

Physics Abstracts

$61.10 \mathrm{D}-75.50 \mathrm{M}$

\title{
Optical scattering on ferrofluid agglomerates
}

\author{
J.-C. Bacri and D. Salin \\ Laboratoire d'Ultrasons (*), Université Pierre et Marie Curie, \\ Tour 13, 4, place Jussieu, 75230 Paris Cedex 05, France
}

(Reçu le 15 juillet 1982, accepté le 23 septembre 1982)

\begin{abstract}
Résumé. - L'observation directe d'un échantillon mince de ferrofluide soumis à un champ magnétique montre l'existence d'un réseau localement hexagonal d'agglomérats. Nous avons observé la figure de diffraction par ce réseau.
\end{abstract}

\begin{abstract}
Direct microscopic observation of a thin sample of ionic ferrofluid, in the presence of a magnetic field, shows the existence of a locally hexagonal lattice of agglomerates. We have observed the unusual pattern of the light scattered by this array.
\end{abstract}

1. Introduction. - Ferrofluids are colloidal suspensions of ferromagnetic grains with a typical size of $100 \AA$. In the presence of a magnetic field, agglomeration phenomena between grains occur [1-3]. The prolate ellipsoid shape [3, 4] of the agglomerates is due to a competition between magnetic energy, which favours elongated agglomerates in the field direction, and surface energy, which favours a spherical shape. Under a given magnetic field the colloidal suspension behaves as an assembly of prolate ellipsoids of nearly the same size and shape [2]. We report here a study of some optical properties of such an assembly of agglomerates when the length $2 a$ of the ellipsoids is limited by the sample glass boundaries.

2. Experimental set up. - We use a ferrofluid obtained through a chemical process without surfactant [5]. The concentration in ionic iron of our ferrofluid suspension is molar. The magneticgrain diameters are nearly $100 \AA$ and the mean distance between grains is $200 \AA$. The ferrofluid sample is put in a disc glass cell of diameter $1 \mathrm{~cm}$. The thicknesses $e$ of our different cells are $20,75,100,200 \mu \mathrm{m}$. The 350 Oersted magnetic field $\mathbf{H}$ is applied along the direction of the cell axis and perpendicular to the glass boundaries (Fig. 1). The incoming laser beam $(\lambda=6328 \AA$, beam diameter $=1 \mathrm{~mm}$ ) has a varying inclination $\theta_{i}$ with the direction of $\mathbf{H}$. We observe, on a screen $\mathbf{P}$ perpendicular to $\mathbf{H}$, the light scattered by the sample. The scattered pattern, in $\mathbf{P}$ at a distance $L=9 \mathrm{~cm}$ from the sample, is analysed using a 3000 ASA Polaroid film.

3. Rough experimental results. - Typical Polaroid pictures of the scattered light are given in four pictures corresponding to the same exposure :

$1 a: \theta_{\mathrm{i}}=0$; the laser beam is in the field direction : we observe a small ring $\mathrm{D}$ centred on the laser beam.

$1 b: \theta_{\mathrm{i}}=2^{\circ}$; the intensity is enhanced on the half ring $\mathrm{D}$ while the other part of the ring vanishes.

(*) Associated with the Centre National de la Recherche Scientifique. 


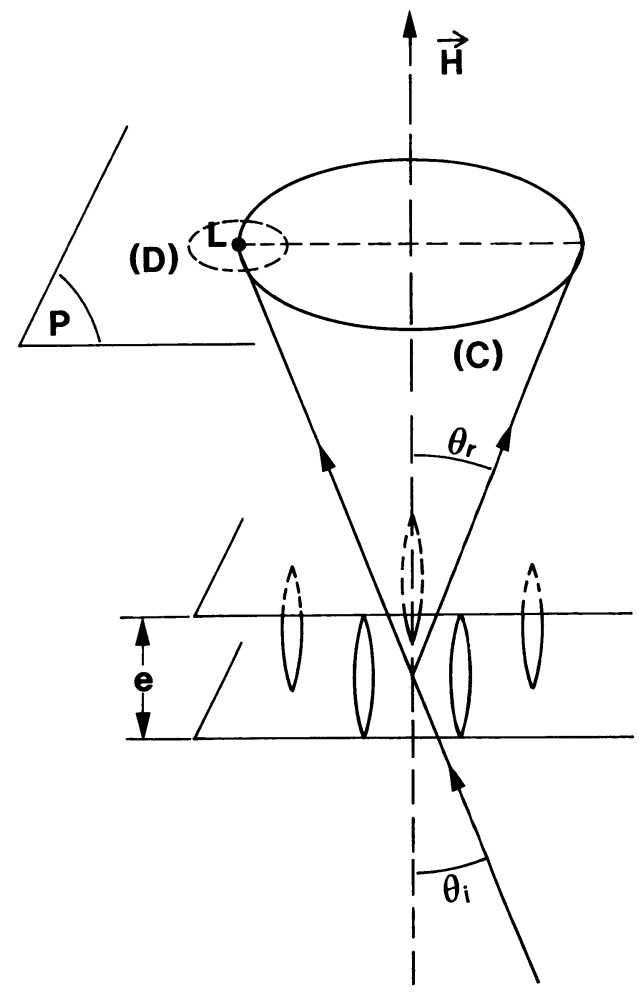

Fig. 1. - Scheme of our experimental set up. $\theta_{\mathrm{i}}$ is the variable angle of the incident laser beam with the field direction. $\mathbf{P}$ is our plane of observation where pictures are taken.

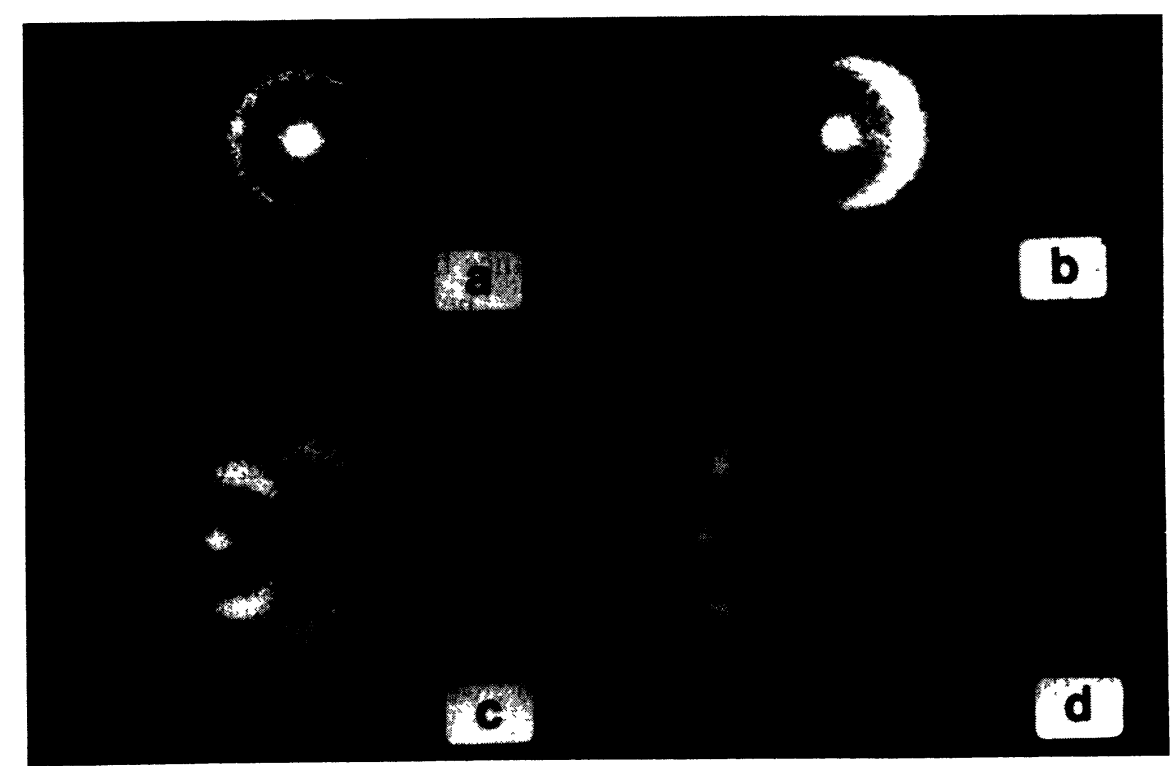

Picture 1. - Pattern of the light scattered by a $75 \mu \mathrm{m}$ thick sample of ferrofluid in presence of a magnetic field for different incident angle $\theta_{\mathrm{i}}$ (Fig. 1) at the same exposure. a) $\left.\left.\left.\theta_{\mathrm{i}}=0 ; b\right) \theta_{\mathrm{i}}=2^{\circ} ; c\right) \theta_{\mathrm{i}}=8^{\circ} ; d\right) \theta_{\mathrm{i}}=15^{\circ}$. 
$1 c: \theta_{\mathrm{i}}=8^{\circ}$; the moon quarter has been transformed into two orange quarters centred on the laser beam. Another ring (ring $\mathrm{C}_{0}$ ) appears centred along the field direction (Fig. 1) and passing through the direct laser beam (L).

$1 d: \theta_{\mathrm{i}}=15^{\circ}$; the initial ring $\mathrm{D}$ has achieved its metamorphosis into two enhancements on ring $\mathrm{C}$, which has become thinner.

Picture $2\left(\theta_{\mathrm{i}}=15^{\circ}, e=100 \mu \mathrm{m}\right)$ is voluntarily overexposed : we observe a series of rings $\mathrm{C}_{n}$ concentric with ring $C_{0}$ with two enhancements on each ring $C_{n}$. These enhancements are all on ring $\mathrm{D}$. The experimental features of ring $\mathrm{C}_{0}$ are :

- for a given $\theta_{\mathrm{i}}$ the radius $R$ of circle $\mathrm{C}_{0}$ is independent of the sample thickness;

- for a given $e, R$ increases rapidly with $\theta_{\mathrm{i}}$, and when $\theta_{\mathrm{i}}$ tends to $\pi / 2$ the circle is transformed into a straight line passing through the direct laser beam $(R=\infty)$. We have measured the angular radius $\theta_{\mathrm{r}}$ (Fig. $1, \operatorname{tg} \theta_{\mathrm{r}}=R / L$ ) versus the incident angle $\theta_{\mathrm{i}}$, for $\theta_{\mathrm{i}}$ from $-\pi / 4$ to $\pi / 4$. We find $\theta_{\mathrm{r}}=\theta_{\mathrm{i}}$ : the $\mathrm{C}_{0}$ ring is the intersection of the plane $\mathrm{P}$ perpendicular to $\mathbf{H}$ with a light-scattered cone of axis $\mathbf{H}$ and generatrix the incident light beam (SL). The last features of ring $\mathrm{C}_{0}$ are that the scattered light has the same polarization as the incident light. At a given $\theta_{i}$ the thickness of the ring decreases as $e$ increases. At a given $e$ the thickness of the ring decreases as $\theta_{\mathrm{i}}$ increases.

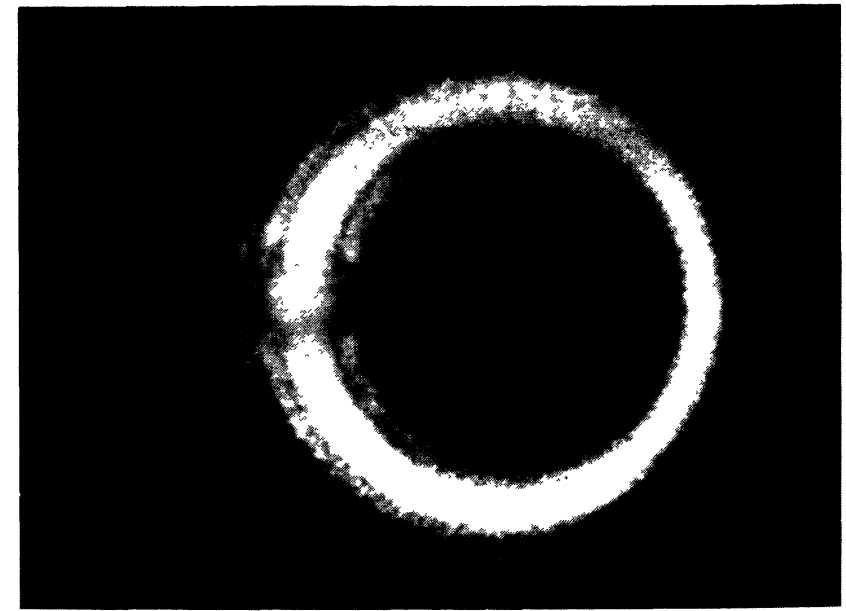

Picture $2 .-\theta_{\mathrm{i}}=15^{\circ}, e=100 \mu \mathrm{m}$. The picture is voluntarily overexposed.

Further analysis of our assembly of agglomerates requires a more direct knowledge of our system. We observed our sample through a microscope in the direction of the field (Picture 3) : the different agglomerates of nearly the same size are arranged on a locally hexagonal array. The mean distances $d$ between nearest neighbours are $d=3,5.5,6.5,9 \mu \mathrm{m}$ for sample thicknesses $e$ of respectively 20,75, 100 and $200 \mu \mathrm{m}$. Picture 3 is a top view of our assembly of agglomerates and we do not know from it whether or not the agglomerates are wetting the glass boundaries. This question is answered by picture 4 which shows the behaviour of agglomerates in a glass microcapillary (diameter $\sim 50 \mu \mathrm{m}$ ). The field is in the direction of the agglomerates. We clearly see that the agglomerates do not wet the surface and appear as elongated needles close to slender ellipsoids. The glass boundaries push them a few microns away from the surface. The diameter $2 b$ of the agglomerates cannot be measured from picture 3 because of optical diffraction. This picture is hazy; the agglomerates are transformed into spherical drops when the field is turned off. From the measured diameter of the spheres and from volume conservation we get

$$
2 b=(1 \pm 0.2) \mu \mathrm{m} \text { for } e=75 \mu \mathrm{m} \text {. }
$$




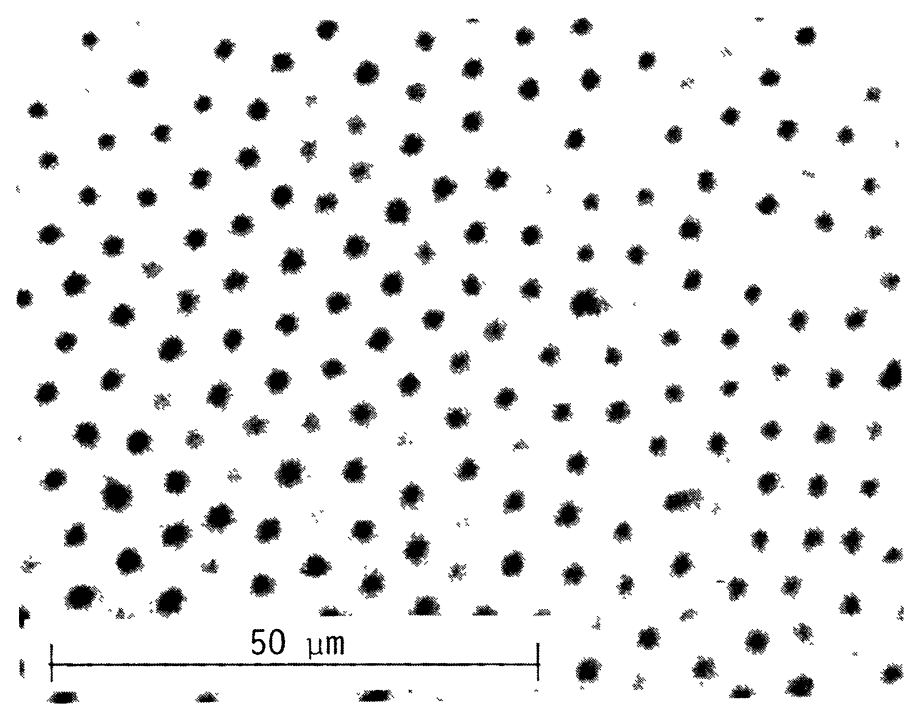

Picture 3. - Microscope picture perpendicular to the field direction of our $75 \mu \mathrm{m}$ sample.

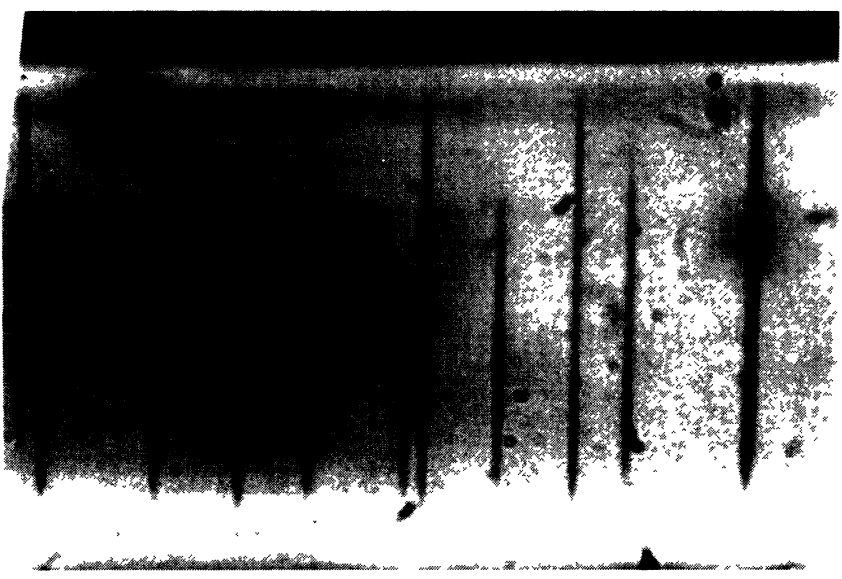

Picture 4. - Microscope picture of agglomerates in a $50 \mu \mathrm{m}$ diameter microcapillary in presence of a magnetic field perpendicular to the tube axis.

As $e$ increases $b$ also increases ( $e=100 \mu \mathrm{m}, 2 b \simeq 1.5 \mu \mathrm{m} ; e=200 \mu \mathrm{m}, 2 b \simeq 3 \mu \mathrm{m}$ ). Physically each agglomerate behaves as a magnetic dipole parallel to the field direction. The hexagonal array is the result of the repulsive dipolar magnetic interaction between different agglomerates. This array is analogous to the two-dimensional colloidal interfacial crystals [6] where the interaction is a dipole-dipole electrostatic interaction.

4. Interpretation. - We will now interpret our optical experiment through an analogy with X-ray diffraction on our sample. For such an analogy, we need the reciprocal lattice [7] of our assembly of agglomerates : from the symmetry of our sample, the reciprocal lattice is one of revolution along the field direction. $\mathbf{k}_{\mathrm{i}}, \mathbf{k}_{\mathrm{r}}, \mathbf{q}$, are the incident, reflected and diffusion wavevectors, $\mathbf{q}=\mathbf{k}_{\mathrm{r}}-\mathbf{k}_{\mathrm{i}} \cdot q_{z}$ and $q_{\mathrm{r}}$ are respectively the components of $\mathbf{q}$ along and perpendicular to $\mathbf{H}$. 
Intensity variations in the reciprocal lattice $\mathcal{R}$ are the result of the form factor $F$ of each individual agglomerate times the interference function $I$ [7] between agglomerates.

We use here the normalized interference function $I_{q=0}=1$, as in [8]. In the case of elongated ellipsoids $(a / b>50)$ the form factor $F$ is close to that of cylinders (radius $b$ and length $2 a)$ :

$$
F=\left(\frac{\sin q_{z} a}{\left(q_{z} a\right)}\right)^{2} \cdot\left(\frac{J_{1}\left(q_{r} b\right)}{\left(q_{r} b\right)}\right)^{2}
$$

$J_{1}$ is a Bessel function. The variation of $F$ in the $q_{z}$ direction is schematized in the insert of figure 2 (at a given $\left.q_{r}\right)$ : the plane $\mathrm{P}_{0}\left(q_{z}=0\right)$ corresponds to a maximum of intensity and secondary maxima are planes $P_{n}$ parallel to $P_{0}$ for wavevectors value $q_{z} \simeq \pm\left(n+\frac{1}{2}\right) \frac{\pi}{a}(n \neq 0$ is a positive

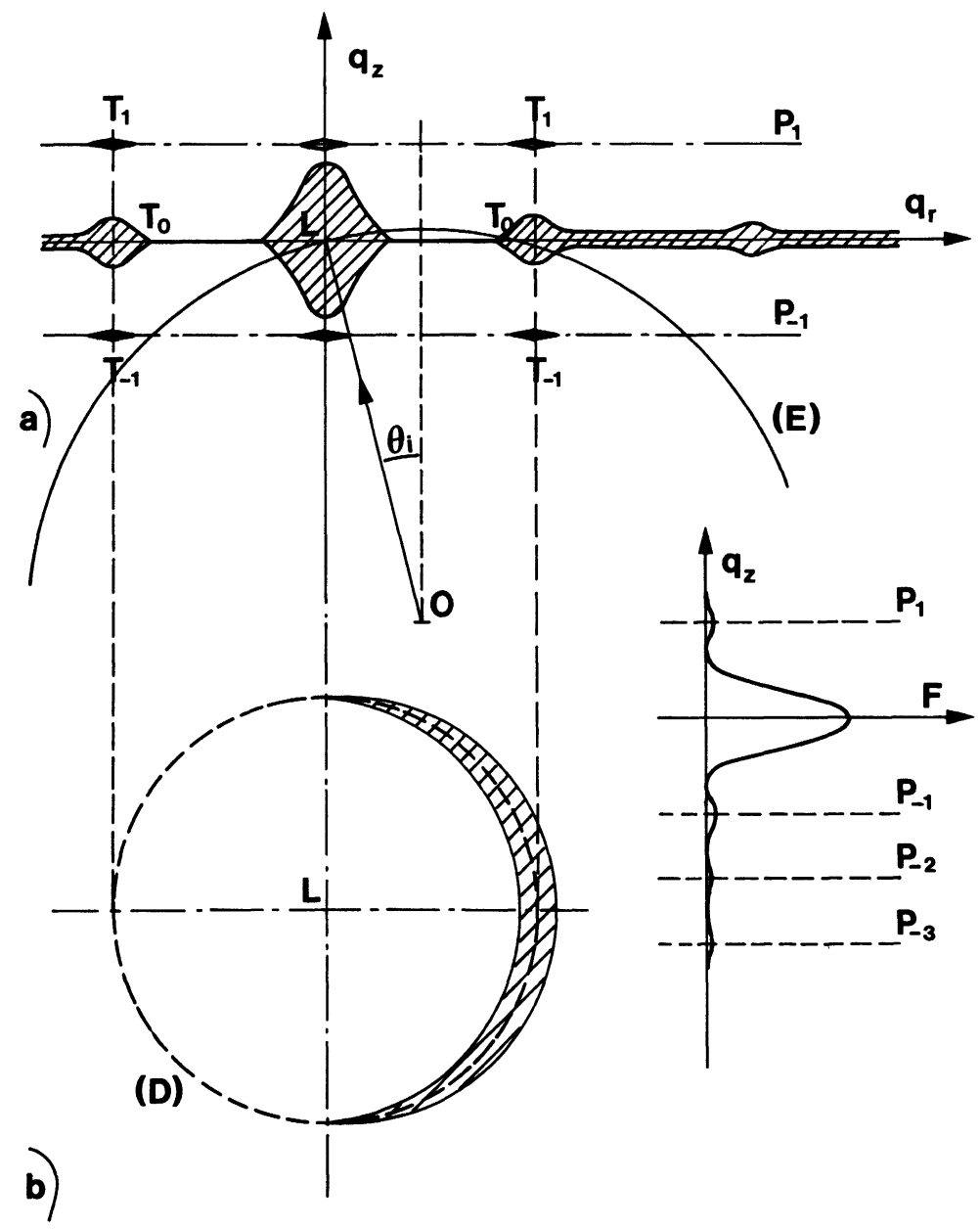

Fig. 2. - a) Scheme of the reciprocal lattice $\mathcal{R}$ of our assembly of agglomerates. $q_{z}$ and $q_{r}$ are the components, along and perpendicular to the field direction, of the diffusion wavevector. Lines limiting dashed surfaces are equal level of intensity lines. $E$ is the Ewald sphere for a small value of the angle $\theta_{i}$ between the laser beam and the field direction. $b$ ) Projection of the intersection of $\mathcal{R}$ and $E$ onto a plane perpendicular to $\mathbf{H}$. This is indeed the light-scattered pattern we observe. Insert : variation of the form factor $F$ with $q_{z}$ at a given $q_{r}$. 
integer). In the $q_{r}$ direction (perpendicular to $\left.\mathbf{H}\right) F$ has its first zero for $q_{r} b \simeq 3.83$. As $b$ is ten times smaller than $d$, for small values of $q_{r}$, intensity variations will be dominated by the effect of the interference function $I$. In the case of a diatomic-molecule gas $I$ is $\left[1+J_{0}\left(q_{r} d\right)\right] / 2(d$ is the interatomic distance) [7]. In our case, with a locally hexagonal array, the interference function given by the seven nearest neighbours is [8] :

$$
I=\left[7+24 J_{0}\left(q_{r} d\right)+6 J_{0}\left(2 q_{r} d\right)+12 J_{0}\left(\sqrt{3} q_{r} d\right)\right] / 49
$$

where $J_{0}$ is a Bessel function and $d$ is the distance between nearest neighbours. $I$ is maximum for $q_{r}=0(I(0)=1)$ and the secondary maximum corresponds to $q_{r} d \simeq 7$. This second maximum is large $(I \sim 0.33)$ and there is a wide deep minimum between this maximum and the direct laser beam $(q=0)$. For large values of $q_{r} d, I$ tends to $1 / 7$. Notice that even for a liquid-like arrangement (only a mean distance $d$ ) the position of the second maximum is close to the value obtained from equation $(2)\left(q_{r} d \sim 7\right)$ but the relative width and depth of the first minimum increases as the long-range order of the lattice increases. The reciprocal lattice is schematized on figures $2 a$ and $3 b$ in a plane $\left(q_{z}, q_{r}\right)$. The line limiting the dashed part of the figure is an (arbitrary) equal level of intensity line of the function $F$ times $I$. The $\mathrm{T}_{n}$ are the tori corresponding to the second maximum

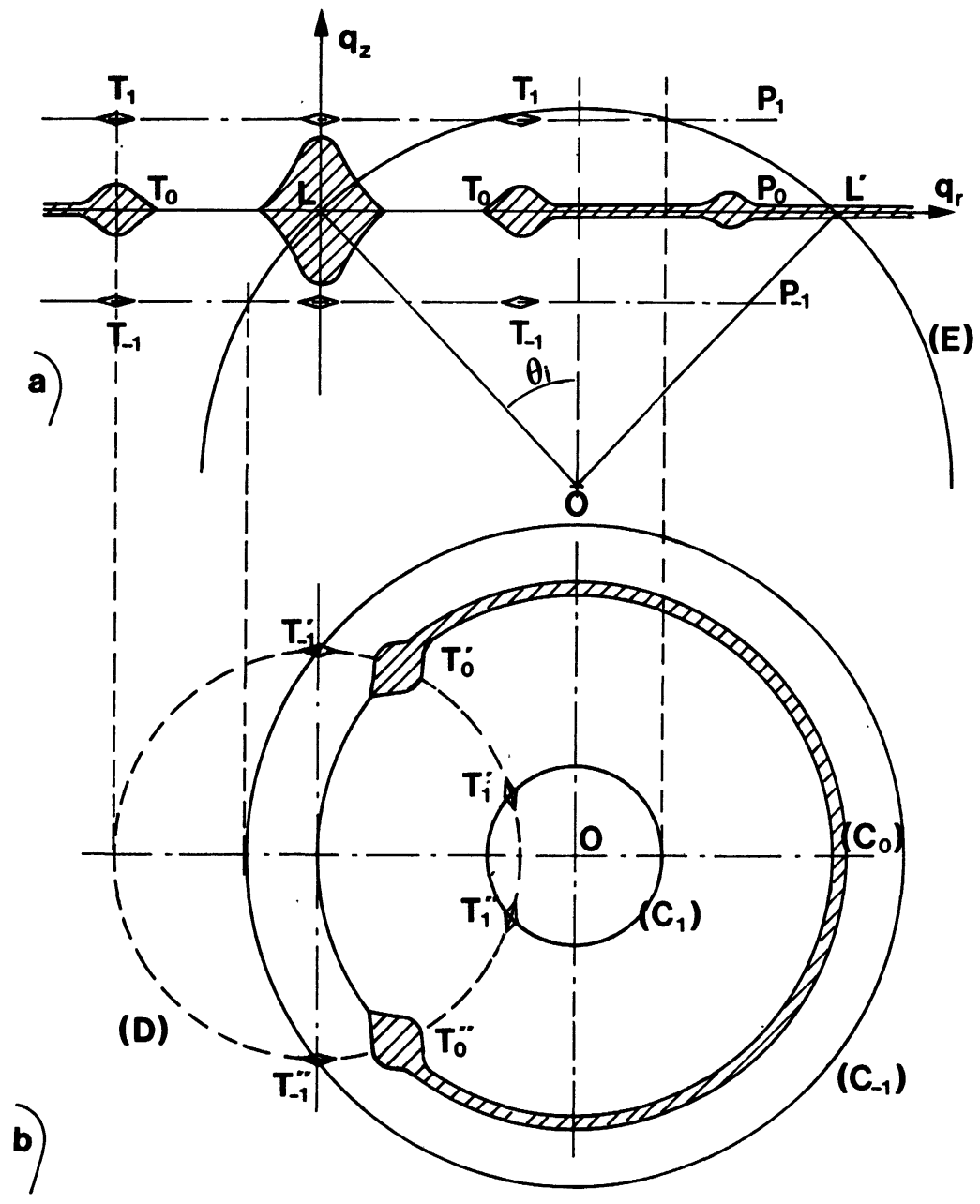

Fig. 3. $-a$ ) and $b$ ) are respectively analogous to figures $2 a$ and $2 b$ but for a much larger incident angle $\theta_{\mathrm{i}}$. 
of the interference function (2). The scattered light from the assembly of agglomerates would be observed for $q$ values to the intersection of the reciprocal lattice $\mathcal{R}$ with the Ewald sphere $\mathrm{E}$ of radius $k=2 \pi / \lambda$, where $\lambda$ is the laser wavelength. (Because we use wavevectors and not wavenumbers, the radius of our Ewald sphere differs by a factor $2 \pi$ from the usual value). $E$ passes by the direct laser spot $\mathrm{L}$ for all values of the angle $\theta_{\mathrm{i}}$. Figures 2 and 3 correpond to two different values of $\theta_{i}$. Figures $2 b$ and $3 b$ are a projection, onto a plane perpendicular to $\mathbf{H}$, of the intersection of $\mathrm{E}$ with $\mathcal{R}$. (These figures correspond to our plane of observation).

Figure 2 : For small values of $\theta_{i}$, due to the $q_{z}$ extension of the torus $T_{0}$, the intersection of $\mathrm{E}$ and $\mathcal{R}$ occurs on a large part of $\mathrm{T}_{0}$. Figure $2 b$ represents in fact picture $1 b$. Picture $1 a$ corresponds to the case $\theta_{i}=0$ where $E$ intersects the torus $T_{0}$ all around (the intensity is smaller than in $1 b)$ : this gives a ring $D$ of diffraction (Debye) of angular radius $\theta_{D}=(6.5 \pm 0.5)^{\circ}$. From the value of $q_{r}$ for the secondary maximum $\left(q_{r} d \sim 7, \sin \theta_{\mathrm{D}}=q_{r} / k\right)$ we get a value $d=(6.2 \pm 0.5) \mu \mathrm{m}$, in reasonable agreement with direct microscope observation $d=(5.5 \pm 0.5) \mu \mathrm{m}(\sim 65$ agglomerates in a $50 \times 50 \mu \mathrm{m}$ area in picture 3). The evolution from pictures $1 a$ to $1 b$ shows that, due to the inclination, a part of ring $D$ has its intensity widely enhanced while the other part vanishes.

Figure 3 : For larger values of $\theta_{i}$ the intersection of $E$ with $\mathcal{R}$ occurs mainly in plane $P_{0}:$ this gives a circle of light $C_{0}$ with two bright spots $T_{0}^{\prime}$ and $T_{0}^{\prime \prime}$ corresponding to the intersection of torus $\mathrm{T}_{0}$ with $\mathrm{E}$. Figure $3 b$ clearly explains pictures $1 c$ and $1 d$. Moreover, the sphere $\mathrm{E}$ also intersects the different planes $P_{n}$ along different circles $C_{n}$ concentric with $C_{0}$ : these circles are visible in the overexposed picture 2 . The two enhancements on each circle $C_{n}$ correspond to the intersection of $\mathrm{C}_{n}$ with $\mathrm{D}$ (points $\mathrm{T}_{n}^{\prime}$ and $\mathrm{T}_{n}^{\prime \prime}$ in figure $3 b$ ). The fact that the different circles $\mathrm{C}_{n}$ are not equidistant is due to the inclination with which $\mathrm{E}$ intersects (Fig. $3 a$ ) the successive planes $\mathrm{P}_{n}$. This also explains why the thickness of ring $\mathrm{C}_{0}$ decreases from picture $1 c$ to $1 d$. More quantitatively, the space between different circle allows a measurement of the length $2 d$ of the agglomerates $\left(q_{z} a= \pm\left(n+\frac{1}{2}\right) \pi\right)$. We get a value $2 a=(90 \pm 10) \mu \mathrm{m}$ in reasonable agreement with the direct microscope observation $(e=2 a)$.

5. Conclusion. - A thin sample of ionic ferrofluid in the presence of a magnetic field (perpendicular to the sample) exhibits an assembly of pseudo-ellipsoidal agglomerates (rich in magnetic particles) arranged on a locally hexagonal lattice. The few-micron dimensions of the array allow a non-usual light scattered pattern. This kind of experiment is a suitable tool for the study of the dynamics of the hexagonal array formation as a function of an applied magnetic field.

\section{References}

[1] Peterson, E. A. and Krueger, D. A., J. Colloid Interface Sci. 62 (1977) 24.

[2] LiaO, W. H. and KRUEGer, D. A., J. Colloid Interface Sci. 70 (1979) 564.

[3] HAYES, C. G., J. Colloid Interface Sci. 52 (1975) 239.

[4] BaCri, J.-C., SAlin, D. and Massart, R., J. Physique-Lett. 43 (1982) L-179.

[5] Massart, R., IEEE Trans. Magn. 17 (1981) 1241.

[6] Pieranski, P., Phys. Rev. Lett. 45 (1980) 569.

[7] GuInIER, A., Théorie et technique de la radiocristallographie (Dunod, Paris) 1956, chapitre 13.

[8] KerKer, M., The Scattering of Light and other Electromagnetic Radiation (Academic Press) 1979. 